\title{
Infection Prevention Strategies in COVID-19 Units Highly Protective While Primary Risks to Healthcare Professionals Come From Coworkers and the Community
}

Shruti Gohil ( $\sim$ skgohil@hs.uci.edu )

UC Irvine Healthcare: UCI Health

\section{Kathleen Quan}

UC Irvine: University of California Irvine

Keith Madey

UC Irvine Healthcare: UCI Health

\section{Suzanne King-Adelsohn}

UC Irvine Healthcare: UCI Health

\section{Tom Tjoa}

UC Irvine: University of California Irvine

Delia Tifrea

UC Irvine Healthcare: UCI Health

\section{Bridgit Crews}

UC Irvine Healthcare: UCI Health

\section{Edwin Monuki}

UC Irvine: University of California Irvine

\section{Saahir Khan}

University of Southern California

\section{Sebastian Schubl}

UC Irvine: University of California Irvine

\section{Cassiana Bittencourt}

UC Irvine Healthcare: UCI Health

Neil Detweiler

UC Irvine Healthcare: UCI Health

\section{Wayne Chang}

UC Irvine Healthcare: UCI Health

Lynn Willis

UC Irvine Healthcare: UCI Health

\section{Usme Khusbu}

UC Irvine Healthcare: UCI Health 
UC Irvine Healthcare: UCI Health

\section{Sherif Rezk}

UC Irvine Healthcare: UCI Health

\section{Cesar Figueroa}

UC Irvine Healthcare: UCI Health

\section{Aarti Jain}

UC Irvine Healthcare: UCI Health

\section{Rafael de Assis}

UC Irvine: University of California Irvine

\section{Philip Felgner}

UC Irvine: University of California Irvine

\section{Robert Edwards}

UC Irvine: University of California Irvine

\section{Lanny Hsieh}

UC Irvine: University of California Irvine

\section{Donald Forthal}

UC Irvine: University of California Irvine

\section{William Wilson}

UC Irvine: University of California Irvine

\section{Michael J Stamos}

UC Irvine: University of California Irvine

\section{Susan Huang}

UC Irvine: University of California Irvine

\section{Research}

Keywords: COVID-19 seroprevalence, healthcare worker COVID-19 exposure, COVID-19 outbreaks, Severe acute respiratory syndrome coronavirus-2, healthcare professional COVID-19 exposure

Posted Date: June 3rd, 2021

DOI: https://doi.org/10.21203/rs.3.rs-566927/v1

License: (-) (i) This work is licensed under a Creative Commons Attribution 4.0 International License. Read Full License 


\section{Abstract}

Background: Early evaluations of healthcare professional (HCP) COVID-19 risk occurred during insufficient personal protective equipment and disproportionate testing, contributing to perceptions of high patient-care related HCP risk. We evaluated HCP COVID-19 seropositivity after accounting for community factors and coworker outbreaks.

Methods: Prior to universal masking, we conducted a single-center retrospective cohort plus cross-sectional study. All HCP 1) seen by Occupational Health for COVID-like symptoms (regardless of test result) or assigned to 2) dedicated COVID-19 units, 3) units with a COVID-19 HCP outbreak, or 4) control units from 01/01/2020-04/15/2020 were offered serologic testing by an FDA-authorized assay plus a research assay against 67 respiratory viruses, including 11 SARS-CoV-2 antigens. Multivariable models assessed the association of demographics, job role, comorbidities, care of a COVID-19 patient, and geocoded socioeconomic status with positive serology.

Results: Of 654 participants, 87 (13.3\%) were seropositive; among these $60.8 \%(\mathrm{~N}=52)$ had never cared for a COVID-19 patient. Being male (OR 1.79, Cl 1.05-3.04, p=0.03), working in a HCP-outbreak unit (OR 2.21, Cl 1.28-3.81, $p<0.01)$, living in a community with low owner-occupied housing ( $O R=1.63, C l=1.00-2.64, p=0.05)$, and ethnically Latino (OR 2.10, $\mathrm{Cl} 1.12-3.96, \mathrm{p}=0.02)$ were positively-associated with COVID-19 seropositivity, while working in dedicated COVID-19 units was negatively-associated (OR $0.53, \mathrm{Cl}=0.30-0.94, \mathrm{p}=0.03)$. The research assay identified 25 additional seropositive individuals (78 [12\%] vs. 53 [8\%], $p<0.01$ ).

Conclusions: Prior to universal masking, HCP COVID-19 risk was dominated by workplace and community exposures while working in a dedicated COVID-19 unit was protective, suggesting that infection prevention protocols prevent patient-to-HCP transmission.

\section{Introduction}

Exposure to transmissible diseases is a known occupational hazard for healthcare professionals (HCPs), which warrants robust infection prevention protocols. Early reports from China demonstrated HCP COVID-19 infection acquisition rates as high as $44 \%$ and subsequent large-scale studies in Spring of 2020 in the UK and U.S. showing a 12-fold higher COVID-19 test-positivity rate among HCPs compared to the community, leading the international healthcare community to adopt robust measures to protect against occupational exposure to COVID-19. ${ }^{1-5}$ Evidence is beginning to emerge that COVID-19 seroprevalence among HCPS mirrors the communities in which they live. ${ }^{6-9}$ Nevertheless the perception of risk among HCPs during patient care remains high and calls into question the effectiveness of infection prevention strategies deployed to protect them. ${ }^{10,11}$

Many currently available studies assess incidence or prevalence of COVID-19 among randomly sampled HCPs and infer occupational risk by defining exposure broadly as having worked in healthcare or in a COVID patient care unit without addressing ongoing clusters (outbreaks) related to coworkers working while ill or community exposures. ${ }^{2,4,5,7-9}$ HCP COVID-19 prevalence may also be exaggerated by testing bias from occupational health screening and ready access to tests. ${ }^{2}$ Finally, HCPs are essential workers that require in- 
person activity, increasing the overall number of both community and work-related interactions within a given day. Epidemiologic studies are needed that assess the added risk borne by HCPs due to COVID-19 patient care compared to in-person coworkers exposures within the healthcare infrastructure and the communities where they live.

In this study, we evaluated whether HCP roles, documented COVID-19 patient care, HCP- outbreak associated coworker exposures, and geocoded community characteristics were associated with the likelihood of polymerase chain reaction (PCR) and serology confirmed COVID-19, enriching for those presenting to Occupational Health $(\mathrm{OH})$ with symptoms, those working in COVID-19-designated care units, those on units with COVID-19 HCP outbreaks, and control units without HCP outbreaks that were non-COVID-19 designated units.

\section{Methods}

\section{Study Design and Population}

We conducted a retrospective cohort study combined with a cross-sectional seroprevalence survey of HCP at an academic medical center in Orange County, California seen by Occupational Health for COVID-19-like symptoms (regardless of COVID-19 PCR result) between January 1, 2020-April 15, 2020. To enrich for COVID19 cases, all HCP assigned to designated COVID-19 units (3), hospital units that experienced a COVID-19 HCP outbreak (3), and matched control units not designated for COVID-19 care and without an active HCP outbreak (3) during the study period were included. HCPs included those involved in direct patient care (e.g. doctors, nurses, nursing assistants, physical/speech/respiratory therapists), and those assigned to the unit for non-patient care duties (e.g. environmental services, pharmacy, dietary, social work, case management). Eligible HCPs were invited to obtain free serologic testing between May 1- June 30, 2020. This study was conducted under hospital operations jointly with approval for the use of a research serologic platform from the University of California Irvine IRB.

\section{Data Collection}

Demographic data and HCP job title/role were obtained from human resources records. Care of an infectious COVID-19 patient or patient room entry was identified using electronic health record (EHR) records from start of the study period through 2 days before COVID-19 serology blood collection. HCPs not identifiable through EHR records (e.g., environmental services) were confirmed by managers to have assigned duty locations during the relevant timeframe. $\mathrm{OH}$ records provided assessment dates for COVID-19 symptoms and PCR results. Epidemiology and Infection Prevention Program records provided details about outbreak investigations and response activities, which included interviews of COVID-19-positive HCP for exposure assessment and evaluations for any linkages between cases by time, location, and activities. In addition, HCP zip codes were geocoded to obtain census-based socioeconomic status (SES) variables focusing on income and housing characteristics (Table 1). ${ }^{12}$ 
Table 1

Characteristics of Healthcare Professional Participants by Serologic Status

\begin{tabular}{|c|c|c|c|c|}
\hline Variable & $\begin{array}{l}\text { All } \\
\text { Participants }\end{array}$ & Seropositive & Seronegative & $\begin{array}{l}\text { P- } \\
\text { Value }\end{array}$ \\
\hline & $N(\%)$ & $N(\%)$ & $N(\%)$ & \\
\hline Total $\mathbf{N}$ & 654 & 87 & 567 & \\
\hline \multicolumn{5}{|l|}{ Invitation Cohort Criteria ${ }^{2}$} \\
\hline Occupational Health Visit & $273(41.7)$ & $52(59.8)$ & $166(29.3)$ & $<.001$ \\
\hline Designated COVID-19 Unit & $275(42.0)$ & $31(35.6)$ & $244(43.0)$ & 0.19 \\
\hline Non-COVID-19 Unit (Control) & $247(37.8)$ & $36(41.4)$ & $211(37.2)$ & 0.46 \\
\hline COVID-19 HCP-Outbreak Unit & $272(41.6)$ & $46(52.9)$ & $226(39.9)$ & 0.02 \\
\hline COVID-19 Non-HCP-Outbreak Unit & $198(30.3)$ & $27(31.0)$ & $171(30.2)$ & 0.86 \\
\hline Age (mean, SD) & 40 & $41(47.1)$ & $40(7.1)$ & 0.24 \\
\hline Male & $181(27.7)$ & $27(31.0)$ & $154(27.2)$ & 0.42 \\
\hline \multicolumn{5}{|l|}{ Race $^{3}$} \\
\hline White & $174(26.6)$ & $21(24.1)$ & $153(27.0)$ & 0.23 \\
\hline Asian & $310(47.4)$ & $39(44.8)$ & $271(47.8)$ & \\
\hline Black & $10(1.5)$ & $2(2.3)$ & $10(1.8)$ & \\
\hline Other & $131(20.0)$ & $17(19.5)$ & $46(8.1)$ & \\
\hline Latino Ethnicity & $101(15.4)$ & $21(24.1)$ & $80(14.1)$ & 0.02 \\
\hline \multicolumn{5}{|l|}{ Socioeconomic Status } \\
\hline $\begin{array}{l}\text { \% Living in zipcodes where } 10 \% \text { or more have } \\
\text { income below poverty line }\end{array}$ & $189(28.9)$ & $27(31.0)$ & $162(28.6)$ & 0.64 \\
\hline $\begin{array}{l}\text { \% Living in zipcodes where } 80 \% \text { or more have } \\
\text { income below median household income }\end{array}$ & $237(36.2)$ & $38(43.7)$ & $199(35.1)$ & 0.12 \\
\hline $\begin{array}{l}\text { \% Living in zipcodes where owner-occupied } \\
\text { household is less than } 50 \%\end{array}$ & $350(53.5)$ & $54(62.1)$ & $296(52.2)$ & 0.09 \\
\hline
\end{tabular}

${ }^{1}$ P-value = chi square comparing seropositive with seronegative. ${ }^{2}$ Participants assigned to cohort if met
criteria at any time during study period and prior to serology sample collection. Occupational Health Visit
$=$ visited occupational health; Designated COVID-19 Unit = HCP (healthcare professional) assigned to
work in designated COVID-19 unit. Non-COVID-19 Unit (Control) = HCP assigned to work in units that did
not admit COVID-19 patients. COVID-19 HCP Outbreak Unit = HCP assigned to work in unit where there
was an ongoing HCP outbreak of COVID-19. COVID-19 Non-HCP Outbreak Unit = HCP assigned to work in
a unit where there was not an active HCP COVID-19 outbreak. ${ }^{3}$ Self-reported on REDCAP survey. ${ }^{4}$ COVID-
19 patient care confirmed by Electronic Health Record (EHR). 


\begin{tabular}{|c|c|c|c|c|}
\hline Variable & $\begin{array}{l}\text { All } \\
\text { Participants }\end{array}$ & Seropositive & Seronegative & $\begin{array}{l}\text { P- } \\
\text { Value }{ }^{1}\end{array}$ \\
\hline $\begin{array}{l}\% \text { Living in zipcodes where } 25 \% \text { or more live in } \\
\text { a house with } 1.5 \text { or more occupants per room }\end{array}$ & $312(47.7)$ & $52(59.8)$ & $260(45.9)$ & 0.02 \\
\hline $\begin{array}{l}\% \text { Living in zipcodes where } 50 \% \text { households } \\
\text { live in structures containing }>=5 \text { units }\end{array}$ & $307(46.9)$ & $42(48.3)$ & $265(46.7)$ & 0.79 \\
\hline Comorbidities (Any) & $141(21.6)$ & $21(24.1)$ & $120(21.2)$ & 0.53 \\
\hline \multicolumn{5}{|l|}{ HCP Role } \\
\hline Registered nurse & $382(58.4)$ & $53(60.9)$ & $329(58.0)$ & 0.12 \\
\hline Nurse Aide & $47(7.2)$ & $12(13.8)$ & $35(6.2)$ & \\
\hline Physical/Occupational Therapist & $12(1.8)$ & $2(2.3)$ & $10(1.8)$ & \\
\hline Respiratory Therapist & $8(1.2)$ & $1(1.1)$ & $7(1.2)$ & \\
\hline Physician & $99(15.1)$ & $7(8.0)$ & $92(16.2)$ & \\
\hline Environmental Services & $12(1.8)$ & $1(1.1)$ & $11(1.9)$ & \\
\hline Other-Direct Patient Care & $33(5.0)$ & $2(2.3)$ & $31(5.5)$ & \\
\hline Not Direct Pt Care & $61(9.3)$ & $9(10.3)$ & $52(9.2)$ & \\
\hline COVID-19 Patient Care (Any) - EHR Review ${ }^{4}$ & $276(42.2)$ & $35(40.2)$ & $241(42.5)$ & 0.69 \\
\hline CoVID-19 Patient Care - Self-Report ${ }^{3}$ & $355(54.3)$ & $41(47.1)$ & $314(55.4)$ & 0.35 \\
\hline Aerosol Generating Procedure ${ }^{3}$ & $97(14.8)$ & $8(9.2)$ & $89(15.7)$ & 0.11 \\
\hline Works in $\mathrm{ICU}^{3}$ & $153(23.4)$ & $19(21.8)$ & $134(23.6)$ & 0.71 \\
\hline \multicolumn{5}{|l|}{ PCR Results } \\
\hline Positive & $54(8.3)$ & $41(47.1)$ & $13(2.3)$ & $<0.01$ \\
\hline Negative & $245(37.5)$ & $19(21.8)$ & $226(39.9)$ & \\
\hline Unknown & $355(54.3)$ & $27(31.0)$ & $328(57.8)$ & \\
\hline Any COVID-19 Symptom on Self-Report ${ }^{3}$ & $395(60.4)$ & $62(71.3)$ & $333(58.7)$ & $<0.01$ \\
\hline No Symptom Reported & $230(35.2)$ & $17(19.5)$ & $213(37.6)$ & \\
\hline
\end{tabular}

${ }^{1} \mathrm{P}$-value $=$ chi square comparing seropositive with seronegative. ${ }^{2} \mathrm{Participants}$ assigned to cohort if met criteria at any time during study period and prior to serology sample collection. Occupational Health Visit = visited occupational health; Designated COVID-19 Unit = HCP (healthcare professional) assigned to work in designated COVID-19 unit. Non-COVID-19 Unit (Control) $=$ HCP assigned to work in units that did not admit COVID-19 patients. COVID-19 HCP Outbreak Unit = HCP assigned to work in unit where there was an ongoing HCP outbreak of COVID-19. COVID-19 Non-HCP Outbreak Unit = HCP assigned to work in a unit where there was not an active HCP COVID-19 outbreak. ${ }^{3}$ Self-reported on REDCAP survey. ${ }^{4}$ COVID19 patient care confirmed by Electronic Health Record (EHR). 


\begin{tabular}{|c|c|c|c|c|}
\hline Variable & $\begin{array}{l}\text { All } \\
\text { Participants }\end{array}$ & Seropositive & Seronegative & $\begin{array}{l}\text { P- } \\
\text { Value }^{1}\end{array}$ \\
\hline \multicolumn{5}{|l|}{ Symptom Type } \\
\hline Non febrile illness & $222(33.9)$ & $19(21.8)$ & $203(35.8)$ & 0.01 \\
\hline Fever & $173(26.5)$ & $43(49.4)$ & $130(22.9)$ & $<0.01$ \\
\hline Fatigue & $234(35.8)$ & 49 (56.3) & $185(32.6)$ & $<0.01$ \\
\hline Chills & $172(26.3)$ & $46(52.9)$ & $126(22.2)$ & $<0.01$ \\
\hline Myalgia & $197(30.1)$ & $47(54.0)$ & $150(26.5)$ & $<0.01$ \\
\hline Congestion & $298(45.6)$ & $42(48.3)$ & $256(45.1)$ & 0.30 \\
\hline Cough & $224(34.3)$ & $37(42.5)$ & $187(33.0)$ & 0.03 \\
\hline Loss of smell & $58(8.9)$ & $30(34.5)$ & $28(4.9)$ & $<0.01$ \\
\hline Shortness of Breath & $106(16.2)$ & $21(24.1)$ & $85(15.0)$ & 0.02 \\
\hline \multicolumn{5}{|c|}{$\begin{array}{l}\text { Days Between Symptoms and Serology } \\
\text { Sample Collection }\end{array}$} \\
\hline$<14$ days & $30(4.6)$ & $4(4.6)$ & $26(4.6)$ & 0.74 \\
\hline 15-29 days & $58(8.9)$ & $10(11.5)$ & $48(8.5)$ & 0.69 \\
\hline $30-44$ days & $17(2.6)$ & $7(8.0)$ & $10(1.8)$ & $<0.01$ \\
\hline $45-59$ days & $107(16.4)$ & $23(26.4)$ & $84(14.8)$ & 0.04 \\
\hline $60-74$ days & $13(2.0)$ & $2(2.3)$ & $11(1.9)$ & 0.67 \\
\hline$>=75$ days & $169(25.8)$ & $15(17.2)$ & $154(27.2)$ & $<0.01$ \\
\hline \multicolumn{5}{|c|}{$\begin{array}{l}{ }^{1} \mathrm{P} \text {-value = chi square comparing seropositive with seronegative. }{ }^{2} \mathrm{P} \text { articipants assigned to cohort if met } \\
\text { criteria at any time during study period and prior to serology sample collection. Occupational Health Visit } \\
=\text { visited occupational health; Designated COVID-19 Unit = HCP (healthcare professional) assigned to } \\
\text { work in designated COVID-19 unit. Non-COVID-19 Unit (Control) = HCP assigned to work in units that did } \\
\text { not admit COVID-19 patients. COVID-19 HCP Outbreak Unit = HCP assigned to work in unit where there } \\
\text { was an ongoing HCP outbreak of COVID-19. COVID-19 Non-HCP Outbreak Unit = HCP assigned to work in } \\
\text { a unit where there was not an active HCP COVID-19 outbreak. }{ }^{3} \text { Self-reported on REDCAP survey. }{ }^{4} \text { COVID- } \\
19 \text { patient care confirmed by Electronic Health Record (EHR). }\end{array}$} \\
\hline
\end{tabular}

Eligible HCP who participated in serologic testing also completed a REDCAP survey requesting demographic information, residential zip code, comorbid conditions, height/weight, COVID-19 patient-care (including aerosol generating procedures (AGP)), and history of COVID-19 symptoms (including type/time of onset). Community COVID prevalence was obtained through the Orange County Health Care Agency. ${ }^{13}$

\section{Laboratory Testing}


Blood samples from participating HCPs were assessed using two platforms, a novel, high- sensitivity Fingerstick Coronavirus Antigen Microarray (COVAM) measuring IgG and IgM antibodies against 67 respiratory viruses, including 11 SARS-CoV-2 antigens, and a Food and Drug Administration Emergency Use Authorized serology assay (FDA-EUA). ${ }^{14-17}$ The primary FDA-EUA assay was the Diazyme SARS-CoV-2 IgG detecting antibodies against SARS-CoV-2 nucleocapsid $(\mathrm{N})$ and spike $(\mathrm{S})$ proteins. ${ }^{17}$ To address potential variability in performance characteristics, results were reflexed for confirmation by an alternate FDA-EUA assay (Beckman Access SARS-CoV-2 IgG assay against S protein or Abbott Architect SARS-CoV-2 IgG assay against the N protein) when Diazyme results were non-reactive in PCR-confirmed or clinically suspected prior SARS-CoV-2 infection. ${ }^{17-20}$ COVID-19 RT-PCR testing was performed using either DiaSorin Molecular Simplexa, m2000 RealTime, or Xpert Xpress SARS-CoV-2. ${ }^{21-23}$ PCR results from other facilities were obtained from $\mathrm{OH}$ records.

\section{Analysis}

Percent participation in the serologic assessment was calculated among each invited cohort. Demographics and characteristics were evaluated as a proportion of participants. Multivariable logistic regression models evaluated the impact of the following variables on the composite outcome of COVID infection defined as either COVID seropositivity (either assay) or PCR positivity: demographics, HCP role, comorbidities, COVID-19 patient care (separately assessed by EHR documentation and survey report), ICU assignment, COVID-19 unit assignment, assignment in a unit during an active HCP outbreak, and geocoded SES variables. Self-reported AGP was collinear with COVID care unit and ICU variables and were not included in this model. SES variables were evaluated among participants according to previously published literature where possible, including: percent living in zipcodes where $10 \%$ or more have income below the poverty line, where $80 \%$ or more have income below the median household income, where $25 \%$ or more live in a house with 1.5 or more occupants per room, and where $50 \%$ households live in structures containing > $=5$ units. ${ }^{24,25}$ We assessed the associated between Latino HCPs and living in areas with higher percentages of Latino residents (above median, 35\%) to assess whether ethnicity can be considered a community-level exposure risk. Percent owneroccupied housing was evaluated using the median cutpoint (58\%) of the dataset since the owner occupancy of Orange County, California is higher than the national average and highly variable due to a large wealth gradient across the county.

Separately, we evaluated symptom association with seropositivity, creating symptom groups using correlation matrices. Symptom groupings were then assessed for an association with seropositivity using multivariable logistic regression. Finally, performance of each serologic assay was compared.

\section{Results}

A total of 1,320 HCPs were invited, including 476 seen by $\mathrm{OH}, 494$ assigned to a designated COVID-19 unit, 388 assigned to a unit that experienced a COVID-19 HCP-outbreak, and 378 assigned to a matched control unit (non-COVID unit and without HCP-outbreak). Some HCPs are counted in multiple categories (e.g. seen by $\mathrm{OH}$ and worked in an HCP-outbreak unit). Among all invited, $654 \mathrm{HCPs}$ participated in serologic testing (623 
completed surveys). Participation was similar between cohorts: OH invited cohort (57.4\% (273/476)), COVID19 designated unit (55.7\% (275/494), COVID-19 HCP-outbreak unit (50.7\% 197/388), and matched nonCOVID-19, non-HCP-outbreak control unit (50.0\% (198/378). Cohort subsets and participant characteristics are provided in Table 1. Compared to all invited HCP, participants with prior COVID-19 PCR-positivity were more likely to participate $(8.3 \%, 54 / 654$ versus $7.0 \%, 92 / 1,320)$, though not statistically significant, $p=0.35$. A total 87 (13.3\%) HCPs were found to be seropositive for COVID-19 by either serology assay. Fourteen specimen tested by FDA-EUA were reflexed to alternate FDA-EUA testing with 2 reactive results. Countywide test-positivity during the study period was $7.2 \%(1,651 / 22,882)$.

On bivariate testing, seropositive and seronegative participants were similar in age, gender, race, comorbidities, HCP role, COVID-19 patient care (EHR or self-report), performance of an AGP, assignment to an ICU, and assignment to a COVID-19-designated units (Table 1). Seropositivity was higher among HCPs seen in $\mathrm{OH}$, prior symptomatic COVID-19, those assigned to a HCP-outbreak unit, Latino ethnicity, and those living in a zip code where $\geq 25 \%$ of residents live in households with more than 1.5 occupants per room (Table 1 ). Notably, $60.8 \%(\mathrm{~N}=52)$ of seropositive HCP never cared for a COVID-19 patient based upon EHR documentation (52.9\% ( $\mathrm{N}=46)$ based upon survey self-report).

The majority $(62 / 87,71.3 \%)$ of seropositive HCPs reported at least one COVID-19 symptom preceding serotesting; most $(47 / 87,54.0 \%)$ had symptoms $\geq 30$ days before testing. Anosmia occurred in $34.5 \%(\mathrm{~N}=30)$ of seropositive compared to $4.9 \%(\mathrm{~N}=28)$ of seronegative HCPs. Congestion was the only symptom not associated with COVID-19 disease in bivariate testing. All PCR-positive participants were seropositive. Latino HCPs were more likely to live in areas with higher Latino populations $(64 \%, N=63 / 98)$ compared to nonLatino HCPs $(44 \%, N=238 / 542, p<0.01$; see Supplemental Table A).

Multivariable regression identified male gender (OR 1.79, $\mathrm{Cl} 1.05-3.04, \mathrm{p}=0.03)$, Latino ethnicity (OR 2.10, Cl $1.12-3.96, p=0.02)$, residence in a community with low owner-occupied housing $(\mathrm{OR}=1.63, \mathrm{Cl}=1.00-2.64, \mathrm{p}$ $=0.05)$, and working in a unit with an HCP outbreak (OR 2.21, Cl 1.28-3.81, p < 0.01) as significantly associated with COVID-19 infection (sero- or PCR-positive) after adjusting for HCP role, comorbidities, and documented COVID-19 patient care (Table 2). Working in a COVID-19 patient unit was associated with a lower likelihood of COVID-19 (OR $0.53, \mathrm{Cl}=0.30-0.94, \mathrm{p}=0.03)$. No other SES variable was significantly associated with COVID-19. 
Table 2

Multivariate Regression Model Evaluating Epidemiologic Risk Factors for Positive Serology or PCR (Composite)

\begin{tabular}{|c|c|c|}
\hline Variable & $\mathrm{OR}(\mathrm{Cl})$ & P-Value \\
\hline Age $^{1}$ (years) & & 0.40 \\
\hline$<35$ & 1.00 & \\
\hline $35-<50$ & $1.53(0.88-2.69)$ & \\
\hline $50-<60$ & $1.68(0.82-3.45)$ & \\
\hline$>60$ & $1.42(0.45-4.44)$ & \\
\hline Male & $1.79(1.04-3.94)$ & 0.03 \\
\hline HCP Role ${ }^{1}$ & & 0.35 \\
\hline Registered nurse & $1.29(0.57-2.95)$ & \\
\hline Nurse Aide & $1.02(0.20-5.74)$ & \\
\hline Physical/Occupational Therapist & $1.05(0.12-9.36)$ & \\
\hline Respiratory Therapist & $0.47(0.20-1.13)$ & \\
\hline Physician & $0.32(0.07-1.43)$ & \\
\hline Environmental Services & $0.19(0.02-1.69)$ & \\
\hline Other-Direct Patient Care & $0.95(0.41-2.19)$ & \\
\hline Not Direct Pt Care & $1.29(0.57-2.95)$ & \\
\hline Comorbidities (Any) & $1.01(0.56-1.79)$ & 0.98 \\
\hline COVID Patient Care by EHR ${ }^{2}$ & $1.10(0.64-1.88)$ & 0.73 \\
\hline Latino Ethnicity ${ }^{3}$ & $2.1(1.12-3.96)$ & 0.02 \\
\hline \% Living in zipcode where $<58 \%$ (median) live in owner-occupied home & $1.63(1.00-2.64)$ & 0.05 \\
\hline Intensive Care Unit & $0.75(0.41-1.37)$ & 0.34 \\
\hline HCP COVID Outbreak Unit & $2.21(1.28-3.81)$ & $<0.01$ \\
\hline Designated COVID Care Unit & $0.53(0.30-0.94)$ & 0.03 \\
\hline
\end{tabular}

Highly correlated symptoms were (rho >0.50) were grouped together: (1) fever, chills, myalgias and (2) cough and congestion. When evaluated in a model adjusting for age, gender, ethnicity, and comorbidities, 
fevers/chills/myalgias (OR 2.00, Cl 1.03-3.90, $\mathrm{p}=0.04$ ) and anosmia (OR 8.81, Cl 4.44-17.50, $\mathrm{p}<0.0001$ ) were associated with COVID-19 (Table 3 ).

Table 3

Multivariate Regression Model Evaluating Clinical Characteristics Associated with Positive Serology or PCR (Composite)

\begin{tabular}{|lll|}
\hline Variable & OR $(\mathrm{Cl})$ & P-Value \\
\hline Age $^{1}$ (years) & & 0.13 \\
\hline$<35$ & 1.00 & \\
\hline $35-<50$ & $1.71(0.91-3.27)$ & \\
\hline $50-<60$ & $2.47(1.13-5.41)$ & 0.18 \\
\hline$>60$ & $1.57(0.42-5.86)$ & 0.03 \\
\hline Male & $1.48(0.84-2.61)$ & 0.69 \\
\hline Latino Ethnicity & $2.06(1.09-3.87)$ & 0.04 \\
\hline Comorbidities (any) & $0.87(0.45-1.69)$ & 0.83 \\
\hline Fever/chills/myalgias ${ }^{2}$ & $2.00(1.02-3.98)$ & $<0.01$ \\
\hline Cough/congestion ${ }^{2}$ & $1.08(0.55-2.39)$ & 0.70 \\
\hline Loss of smell & $8.81(4.43-17.59)$ & \\
\hline Shortness of breath & $0.88(0.44-1.74)$ & \\
\hline${ }^{1}$ Referent for Age = $<35$ years. ${ }^{2}$ Symptoms highly correlated $(>0.50)$ with each other and evaluated as a \\
\hline composite.
\end{tabular}

The COVAM assay identified 25 additional seropositive individuals (78 [12\%]) compared to the FDA-EUA assay $(53[8 \%]))$ (Table 4), $p<0.01$. The FDA-EUA assay had a higher (but not statistically significant) proportion of seropositive HCPs who reported any COVID-19 symptoms, specifically fever, chills, myalgias, fatigue, anosmia, cough, and shortness of breath, while COVAM had a higher proportion of seropositive HCPs who were asymptomatic, had only non-febrile illness, or symptoms $>75$ days prior to testing. When restricting to the 41 PCR-confirmed participants (Table 5), all were seropositive by the COVAM assay while 38 (92.7\%) were seropositive by the FDA-EUA assay; among the two additional patients detected by COVAM, both had symptoms more than 60 days before blood draw. 
Table 4

Comparative Evaluation of FDA-EUA versus COVAM Serology by Symptom Characteristics

Symptom Characteristics By Serology Assay

Percent Seropositive Within Each Symptom Category

FDA-EUA $\quad \mathrm{N}(\%)$ of
Assay or
Microarray

$N(\%)$ of

COVAM

Serology with

$\begin{array}{ll}\text { p- } & \mathrm{N}(\%) \text { of } \\ \text { value } & \begin{array}{l}\text { Characteristic } \\ \text { with Positive } \\ \text { FDA-EUA } \\ \text { Serology }\end{array}\end{array}$

$\mathrm{N}(\%)$ of Characteristic

FDA-EUA

Serology with

\section{Characteristic}

Seropositive 87

- Total

53

78

$<60.9 \%$

$89.7 \%$

Reported to

Occupational

$67(77.0) \quad 46(86.8)$

$63(80.8)$

0.01

Characteristic

with Positive

COVAM

Serology

Health

$\begin{array}{lllllll}\text { Any } & 62(71.3) & 42(79.2) & 58(74.4) & \text { NS } & 67.7 \% & 93.5 \% \\ \text { Symptom } \\ \text { Reported }\end{array}$

\section{No Symptom
Reported
Symptom
Type}

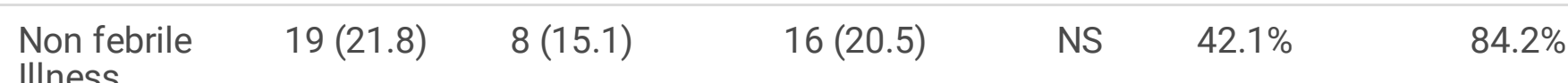

\begin{tabular}{lllllll} 
Fever & $43(49.4)$ & $34(64.2)$ & $42(53.8)$ & NS & $79.1 \%$ & $97.7 \%$ \\
Fatigue & $49(56.3)$ & $35(66.0)$ & $46(59.0)$ & NS & $71.4 \%$ & $93.9 \%$ \\
Chills & $46(52.9)$ & $34(64.2)$ & $43(55.1)$ & NS & $73.9 \%$ & $93.5 \%$ \\
Myalgia & $47(54.0)$ & $33(62.3)$ & $45(57.7)$ & NS & $70.2 \%$ & $95.7 \%$ \\
Congestion & $42(48.3)$ & $25(47.2)$ & $39(50.0)$ & NS & $59.5 \%$ & $92.9 \%$ \\
$\begin{array}{l}\text { Cough } \\
\begin{array}{l}\text { Loss of } \\
\text { smell }\end{array}\end{array}$ & $37(42.5)$ & $28(52.8)$ & $34(43.6)$ & NS & $75.7 \%$ & $91.9 \%$ \\
\hline
\end{tabular}

${ }^{1} \mathrm{P}$-value $=$ chi square comparing FDA-EUA with COVAM serology. Abbreviations: FDA-EUA $=$ Food and Drug Administration Emergency Use Authorization; COVAM = Coronavirus Antigen Microarray 


\begin{tabular}{|c|c|c|c|c|c|c|}
\hline \multirow[b]{2}{*}{$\begin{array}{l}\text { Shortness of } \\
\text { Breath }\end{array}$} & \multicolumn{4}{|c|}{ Symptom Characteristics By Serology Assay } & \multicolumn{2}{|c|}{$\begin{array}{l}\text { Percent Seropositive Within } \\
\text { Each Symptom Category }\end{array}$} \\
\hline & $21(24.1)$ & $16(30.2)$ & $19(24.4)$ & NS & $76.2 \%$ & $96.3 \%$ \\
\hline $\begin{array}{l}\text { Days } \\
\text { Between } \\
\text { Symptoms } \\
\text { and Serology } \\
\text { Sample } \\
\text { Collection }\end{array}$ & & & & NS & & \\
\hline$<14$ days & $4(4.6)$ & $3(5.7)$ & $4(5.1)$ & & $75.0 \%$ & $100.0 \%$ \\
\hline 15-29 days & $10(11.5)$ & $8(15.1)$ & $9(11.5)$ & & $80.0 \%$ & $90.0 \%$ \\
\hline $30-44$ days & $7(8.0)$ & $7(13.2)$ & $7(9.0)$ & & $100.0 \%$ & $100.0 \%$ \\
\hline $45-59$ days & $23(26.4)$ & $19(35.8)$ & $23(29.5)$ & & $82.6 \%$ & $100.0 \%$ \\
\hline $60-74$ days & $2(2.3)$ & $1(1.9)$ & $2(2.6)$ & & $50.0 \%$ & $100.0 \%$ \\
\hline$>=75$ days & $15(17.2)$ & $4(7.5)$ & $12(15.4)$ & & $26.7 \%$ & $80.0 \%$ \\
\hline
\end{tabular}


Table 5

Comparison of FDA-EUA and COVAM Seropositivity and Symptom Characteristics Among those with PCRConfirmed COVID

\begin{tabular}{|c|c|c|c|c|c|c|c|}
\hline \multirow{2}{*}{$\begin{array}{l}\text { Seropositive and PCR-positives } \\
\text { PCR-positive - Total }\end{array}$} & \multicolumn{2}{|c|}{$\begin{array}{l}\text { FDA-EUA Assay or } \\
\text { COVAM N(\%) }\end{array}$} & \multicolumn{2}{|c|}{$\begin{array}{l}\text { FDA-EUA Assay } \\
\text { Serology N (\%) }\end{array}$} & \multicolumn{2}{|c|}{$\begin{array}{l}\text { COVAM } \\
\text { Serology N } \\
\text { (\%) }\end{array}$} & \multirow[t]{2}{*}{$\begin{array}{l}\text { P- } \\
\text { value }\end{array}$} \\
\hline & 41 & & 38 & & 41 & & \\
\hline Seropositive & 41 & & 38 & & 41 & & \\
\hline Reported to Occ Health & 41 & & 38 & & 41 & & \\
\hline No Symptom Reported & 4 & $9.8 \%$ & 4 & $10.5 \%$ & 4 & $9.8 \%$ & 0.81 \\
\hline Any Symptom Reported & 37 & $90.2 \%$ & 34 & $89.5 \%$ & 37 & $90.2 \%$ & 0.81 \\
\hline \multicolumn{8}{|l|}{ Symptom Type } \\
\hline Fever & 31 & $75.6 \%$ & 30 & $78.9 \%$ & 31 & $75.6 \%$ & 0.77 \\
\hline Non febrile Illness & 6 & $14.6 \%$ & 4 & $10.5 \%$ & 6 & $14.6 \%$ & 0.84 \\
\hline Fatigue & 31 & $75.6 \%$ & 29 & $76.3 \%$ & 31 & $75.6 \%$ & 0.89 \\
\hline Chills & 30 & $73.2 \%$ & 28 & $73.7 \%$ & 30 & $73.2 \%$ & 0.90 \\
\hline Myalgia & 30 & $73.2 \%$ & 28 & $73.7 \%$ & 30 & $73.2 \%$ & 0.90 \\
\hline Congestion & 21 & $51.2 \%$ & 19 & $50.0 \%$ & 21 & $51.2 \%$ & 0.94 \\
\hline Cough & 24 & $58.5 \%$ & 23 & $60.5 \%$ & 24 & $58.5 \%$ & 0.91 \\
\hline Loss of smell & 27 & $65.9 \%$ & 26 & $68.4 \%$ & 27 & $65.9 \%$ & 0.87 \\
\hline Shortness of Breath & 15 & $36.6 \%$ & 14 & $36.8 \%$ & 15 & $36.6 \%$ & 0.93 \\
\hline \multicolumn{8}{|l|}{$\begin{array}{l}\text { Days Between Symptoms and } \\
\text { Serology Sample Collection }\end{array}$} \\
\hline$<14$ days & 4 & $9.8 \%$ & 3 & $7.9 \%$ & 4 & $9.8 \%$ & 0.96 \\
\hline 15-29 days & 7 & $17.1 \%$ & 6 & $15.8 \%$ & 7 & $17.1 \%$ & \\
\hline 30-44 days & 7 & $17.1 \%$ & 7 & $18.4 \%$ & 7 & $17.1 \%$ & \\
\hline 45-59 days & 17 & $41.5 \%$ & 16 & $42.1 \%$ & 17 & $41.5 \%$ & \\
\hline 60-74 days & 1 & $2.4 \%$ & 1 & $2.6 \%$ & 1 & $2.4 \%$ & \\
\hline >=75 days & 1 & $2.4 \%$ & 1 & $2.6 \%$ & 1 & $2.4 \%$ & \\
\hline
\end{tabular}

During the study period, which occurred before implementation of universal masking and before mandatory N95 use, there were three units with HCP-outbreaks involving a total of 18 HCPs; of these, 8 (44.4\%) were exposed to an ill coworker, 6 (33.3\%) had no known exposure source, and $3(16.7 \%)$ had a community 
exposure source. Only 1 (5.6\%) HCP infection was plausibly related to patient exposure due to breach of personal protective equipment (PPE). The first outbreak began with an HCP who had traveled to an area with widespread COVID-19 and had never cared for a COVID-19 patient; this HCP developed muscle and joint pains within the incubation period while at work followed by a sore throat the next day, prompting symptom report and testing. While symptomatic, the HCP interacted directly with two other HCP who subsequently developed COVID-19 within 4-5 days. Their interactions involved hand-off of a nursing cell phone and sharing lunch in a breakroom.

The second outbreak began with a HCP who had no clear source for COVID-19 at work or in the community. The HCP developed symptoms while working and likely infected three other coworkers who worked the same shift. These HCPs also developed symptoms while working and resulted in a cascade of four additional COVID-19 infections in HCPs who worked during the same shift and/or shared spaces (e.g., breakroom, nursing station, skills class). One physician who did not regularly work on the unit spent less than 1 hour reviewing charts at the nursing station and developed COVID-19 without having entered a COVID-19 patient room.

In the third outbreak, a potluck led to 6 HCPs developing COVID-19. Preceding the potluck, a patient tested positive for COVID-19 after being unrecognized while admitted. This patient underwent emergent resuscitation and intubation before COVID-19 diagnosis, but none of the code blue providers developed COVID-19. Five of the six HCPs had not provided care for a COVID-19 patient in the weeks prior to developing symptoms. The last HCP was an administrative staff that assisted with obtaining supplies during the code blue and did not have direct patient contact; based on exposure history, this HCP most likely acquired illness from symptomatic coworkers in the nursing station.

\section{Discussion}

Appropriate attribution of true HCP exposure risk must be contextualized by the exposure sources faced in community settings and non-patient care work activities, while simultaneously accounting for the infection prevention strategies in place within healthcare settings to mitigate patient exposures. The top predictors of COVID-19 seropositivity were working in an HCP-outbreak unit, Latino ethnicity, and living in zip code with lower owner-occupied housing, reflecting the important contribution of coworkers and community exposures above and beyond documented COVID-19 patient care. In fact, we found that working in a COVID-19 unit (with contact, eye, and droplet-based mask precautions) was protective against COVID-19, after accounting for the above community and work-related factors, including HCP role and documented care of a COVID-19 patient, suggesting that infection prevention protocols and practices are highly effective in preventing patient-to-HCP transmission.

The majority of HCP workhours are spent performing indirect patient care tasks, such as charting, rounding, or discussing and coordinating patient care. Direct patient-facing care constitutes $20-40 \%$ of HCP time; in contact precautions rooms, this time decreases by $18 \% .{ }^{26-28}$ In the setting of a pandemic, heightened awareness of personal risk during COVID-19 patient care results in high compliance with PPE and hand hygiene, further decreasing the likelihood of direct patient care exposures. ${ }^{29-32} \mathrm{HCPs}$ spend comparatively 
more time in communal settings, often in confined and shared spaces such as nursing stations, physician workrooms, breakrooms, and conference rooms. ${ }^{33,34}$ Further exacerbating coworker exposure risk is the propensity to work while actively ill, an unintended consequence of strong work ethics that lead to working long hours despite physical discomfort or sickness. ${ }^{35}$

Our finding that $60 \%$ of HCPs involved in COVID-19 outbreaks never cared for a COVID-19 patient and that HCP infections appeared to propagate between coworkers highlights the importance of robust daily symptom screening, enforcement of working well policies, and strict compliance with universal masking and social distancing in communal spaces. Current regulatory agencies emphasize high standards for hand hygiene, PPE compliance, and environmental cleaning practices for patient safety; translating these standards to shared HCP spaces and activities is imperative, particularly during a pandemic. ${ }^{36,37}$ This includes increasing the number and strategic placement of hand hygiene stations, environmental cleaning products for high touch items in workstations and breakrooms, workflow and structural modifications to minimize crowding, and robust systems-level enforcement of HCP compliance with protocols.

COVID-19 cases in our county were higher among Latino and densely populated communities, mimicking national trends showing disproportionately greater burden in socioeconomically disadvantaged areas. ${ }^{13,24}$ COVID-19 exposures are more likely in communities with more household crowding, low-wage essential workers, and reliance on public transportation, increasing the likelihood of encountering crowded conditions. ${ }^{24,38}$ This has two important implications for hospital pandemic response. First, infection prevention strategies to reduce HCP risk should also address community level risks within the workforce, targeting outreach to HCPs from high risk communities, providing culturally and linguistically appropriate education on how to minimize exposure risks both within healthcare and community settings. Since HCPs in socioeconomically disadvantaged communities live with household members who also carry heightened risk for acquiring infection, assuring HCPs have an understanding of home-based household infection prevention protocols may afford additional protection. Second, essential workplaces must partner with public health to educate, support contact tracing and quarantining strategies, and facilitate timely testing and management in high risk communities where their workers reside.

HCP perception of risk is integral to promoting behaviors that reduce exposure risk. Fear and concern about acquiring COVID-19 results in high adherence to hand hygiene and PPE during patient interactions. ${ }^{30}$ In contrast, HCPs perceive coworkers or community exposures as less risky, which can increase transmission opportunities in common spaces and breakrooms. The earliest reports of high COVID-19 risk among HCPs occurred in the setting of inadequate PPE and nascent infection prevention protocols. 1,3,30 Subsequently, national and state regulatory bodies, nursing unions, and the media assumed inadequate PPE was the primary exposure risk in healthcare settings, missing important contributions from coworker or community risk. This led to increasingly intensive direct-care PPE requirements without equal attention to the contribution of coworker and community exposures. Notably, the outbreaks in this study occurred before N95 requirements for patient care and we found that droplet-based PPE successfully prevented COVID transmission from patients-to-HCP, while coworker-to-coworker exposures were the sources of unit HCP outbreaks. 
This study allowed comparison between the FDA-EUA assays and a novel microarray assay capable of differentiating 67 separate respiratory virus antigen, including influenza and four common cold coronaviruses. ${ }^{15}$ Though we found high concordance between the two assays, the microarray assay identified additional individuals who had asymptomatic or non-febrile infection, or illness beyond 75 days prior to testing. The microarray assay could help differentiate between COVID-19 and other respiratory viruses, which could be particularly helpful during the cold and flu season.

Our findings are limited by a single institutional experience; however infection prevention programs across the nation report similar experiences with coworker-associated COVID-transmissions. Second, our county has an overall higher per capita income compared to other counties although the wealth gradient across the county is notable and able to identify SES-based risk factors. Third, participation was voluntary which could introduce sampling bias, although participation across the invited cohorts were similar.

HCP COVID-19 exposure risks must be evaluated and interpreted within the full context of workplace and community exposure sources. When accounting for the protections in place for direct patient care activities, workplace and community exposures appear to dominate COVID-19 risk. Additional investments are needed to account for familiar behaviors and shared meals among co-workers and improve infection prevention strategies to reduce transmission. In addition, extensive investment is needed to target pandemic response efforts to lower socioeconomic status communities where essential workers live. Healthcare systems should consider opportunities to partner with public health to leverage COVID-prevention expertise to these areas.

\section{Declarations}

\section{Declarations}

(1) Ethics approval and consent to participate: This study was conducted under hospital operations jointly with approval for the use of a research serologic platform from the University of California Irvine IRB. (2) Consent for publication - all authors have consented to publication. (3) Availability of data and materials all data are available to authors and subject to health information protections. (4) Conflicts of Interest - The authors report no relevant financial or other disclosures. (5) Funding - UC Irvine School of Medicine research funding award. (6) Authors' contributions - all authors have contributed to the work sufficient to be included on this manuscript. (7) Acknowledgements - none.

\section{References}

1. Zhou Q, Gao Y, Wang X, et al. Nosocomial infections among patients with COVID-19, SARS and MERS: a rapid review and meta-analysis. Ann Transl Med. 2020;8:629.

2. Nguyen LH, Drew DA, Graham MS, et al. Risk of COVID-19 among front-line health-care workers and the general community: a prospective cohort study. Lancet Public Health. 2020;5:e475-e83.

3. Zhang Z, Liu S, Xiang M, et al. Protecting healthcare personnel from 2019-nCoV infection risks: lessons and suggestions. Front Med. 2020;14:229-31. 
4. Mutambudzi M, Niedwiedz C, Macdonald EB, et al. Occupation and risk of severe COVID-19: prospective cohort study of 120075 UK Biobank participants. Occup Environ Med 2020.

5. Barrett ES, Horton DB, Roy J, et al. Prevalence of SARS-CoV-2 infection in previously undiagnosed health care workers in New Jersey, at the onset of the U.S. COVID-19 pandemic. BMC Infect Dis. 2020;20:853.

6. Kluytmans-van den Bergh MFQ, Buiting AGM, Pas SD, et al. Prevalence and Clinical Presentation of Health Care Workers With Symptoms of Coronavirus Disease 2019 in 2 Dutch Hospitals During an Early Phase of the Pandemic. JAMA Netw Open. 2020;3:e209673.

7. Ran L, Chen X, Wang Y, Wu W, Zhang L, Tan X. Risk Factors of Healthcare Workers With Coronavirus Disease 2019: A Retrospective Cohort Study in a Designated Hospital of Wuhan in China. Clin Infect Dis. 2020;71:2218-21.

8. Baker JM, Nelson KN, Overton E, et al. Quantification of Occupational and Community Risk Factors for SARS-CoV-2 Seropositivity Among Health Care Workers in a Large U.S. Health Care System. Ann Intern Med; 2021.

9. Jacob JT, Baker JM, Fridkin SK, et al. Risk Factors Associated With SARS-CoV-2 Seropositivity Among US Health Care Personnel. JAMA Netw Open. 2021;4:e211283.

10. Doraiswamy PM, Chilukuri MM, Ariely D, Linares AR. Physician Perceptions of Catching COVID-19: Insights from a Global Survey. J Gen Intern Med 2021.

11. Yuki Sahashi HE, Tadafumi Sugimoto T, Nabeta K, Nishizaki A, Kikuchi S, Matsumoto H, Sato T, Goto, Kohei Hasegawa, Yuya Matsue Worries and concerns among healthcare workers during the coronavirus 2019 pandemic: A web-based cross-sectional survey. Nature: Humanities and Social Sciences Communications 2021;8, 41 (2021).

12. US Census Bureau. (Accessed. October, 2020, at https://www.census.gov/data.html.).

13. Orange County COVID-19 Dashboard. (Accessed February 5. 2021, at https://occovid19.ochealthinfo.com/coronavirus-in-oc.).

14. Khan S, Nakajima R, Jain A, et al. Analysis of Serologic Cross-Reactivity Between Common Human Coronaviruses and SARS-CoV-2 Using Coronavirus Antigen Microarray. bioRxiv 2020.

15. de Assis RR, Jain A, Nakajima R, et al. Analysis of SARS-CoV-2 antibodies in COVID-19 convalescent blood using a coronavirus antigen microarray. Nat Commun. 2021;12:6.

16. Assis R, Jain A, Nakajima R, et al. Distinct SARS-CoV-2 antibody reactivity patterns in coronavirus convalescent plasma revealed by a coronavirus antigen microarray. Sci Rep. 2021;11:7554.

17. Food \& Drug Administration COVID-19 EUA Authorized Serology Test Performance. (Accessed May 1. 2021, at https://www.fda.gov/medical-devices/coronavirus-disease-2019-covid-19-emergency-useauthorizations-medical-devices/eua-authorized-serology-test-performance.).

18. Suhandynata RT, Hoffman MA, Kelner MJ, McLawhon RW, Reed SL, Fitzgerald RL. Multi-Platform Comparison of SARS-CoV-2 Serology Assays for the Detection of COVID-19. J Appl Lab Med. 2020;5:1324-36.

19. Yin L, Tang Y, Chen X, Sun Y. Measurement Differences Between Two Immunoassay Systems for LH and FSH: A Comparison of Roche Cobas e601 vs. Abbott Architect i2000sr. Clin Lab. 2018;64:295-301. 
20. Ward MD, Mullins KE, Pickett E, et al. Performance of four automated SARS-CoV-2 serology assay platforms in a large cohort including susceptible COVID-19 negative and COVID-19 positive patients. J Appl Lab Med 2021.

21. Food \& Drug Administration In Vitro Diagnostics EUAs - Molecular Diagnostic Tests for SARS-CoV-2. (Accessed May 1. 2021, at https://www.fda.gov/medical-devices/coronavirus-disease-2019-covid-19emergency-use-authorizations-medical-devices/in-vitro-diagnostics-euas-molecular-diagnostic-testssars-cov-2.).

22. Loeffelholz MJ, Alland D, Butler-Wu SM, et al. Multicenter Evaluation of the Cepheid Xpert Xpress SARSCoV-2 Test. J Clin Microbiol 2020;58.

23. Degli-Angeli E, Dragavon J, Huang ML, et al. Validation and verification of the Abbott RealTime SARSCoV-2 assay analytical and clinical performance. J Clin Virol. 2020;129:104474.

24. Scannell Bryan M, Sun J, Jagai J, et al. Coronavirus disease 2019 (COVID-19) mortality and neighborhood characteristics in Chicago. Ann Epidemiol 2021;56:47-54 e5.

25. Niedzwiedz CL, O'Donnell CA, Jani BD, et al. Ethnic and socioeconomic differences in SARS-CoV-2 infection: prospective cohort study using UK Biobank. BMC Med. 2020;18:160.

26. Alromaihi D, Godfrey A, Dimoski T, Gunnels P, Scher E, Baker-Genaw K. Internal medicine residents' time study: paperwork versus patient care. J Grad Med Educ. 2011;3:550-3.

27. Westbrook JI, Duffield C, Li L, Creswick NJ. How much time do nurses have for patients? A longitudinal study quantifying hospital nurses' patterns of task time distribution and interactions with health professionals. BMC Health Serv Res. 2011;11:319.

28. Morgan DJ, Pineles L, Shardell M, et al. The effect of contact precautions on healthcare worker activity in acute care hospitals. Infect Control Hosp Epidemiol. 2013;34:69-73.

29. Israel S, Harpaz K, Radvogin E, et al. Dramatically improved hand hygiene performance rates at time of coronavirus pandemic. Clin Microbiol Infect. 2020;26:1566-8.

30. Liu M, Cheng SZ, Xu KW, et al. Use of personal protective equipment against coronavirus disease 2019 by healthcare professionals in Wuhan, China: cross sectional study. BMJ. 2020;369:m2195.

31. Neuwirth MM, Mattner F, Otchwemah R. Adherence to personal protective equipment use among healthcare workers caring for confirmed COVID-19 and alleged non-COVID-19 patients. Antimicrob Resist Infect Control. 2020;9:199.

32. Baker MA, Rhee C, Fiumara K, et al. COVID-19 infections among HCWs exposed to a patient with a delayed diagnosis of COVID-19. Infect Control Hosp Epidemiol. 2020;41:1075-6.

33. Ellsworth M, Chang M, Ostrosky-Zeichner L. Mind the gap: The hospital breakroom. Am J Infect Control. 2020;48:1285.

34. Butler R, Monsalve M, Thomas GW, et al. Estimating Time Physicians and Other Health Care Workers Spend with Patients in an Intensive Care Unit Using a Sensor Network. Am J Med. 2018;131:972. e9e15.

35. Szymczak JE, Smathers S, Hoegg C, Klieger S, Coffin SE, Sammons JS. Reasons Why Physicians and Advanced Practice Clinicians Work While Sick: A Mixed-Methods Analysis. JAMA Pediatr. 2015;169:815-21. 
36. Infection Prevention and Control. (Accessed April 26. 2021, at https://www.jointcommission.org/resources/patient-safety-topics/infection-prevention-and-control/.).

37. Occupational Safety and Health Administration, Healthcare Infectious Diseases. (Accessed April 26. 2021, at https://www.osha.gov/healthcare/infectious-diseases.).

38. Jay J, Bor J, Nsoesie EO, et al. Neighbourhood income and physical distancing during the COVID-19 pandemic in the United States. Nat Hum Behav. 2020;4:1294-302.

\section{Supplementary Files}

This is a list of supplementary files associated with this preprint. Click to download.

- SupplementalTableA.docx 